\title{
TENGO INTENCIÓN DE HACERME UNA MAMOGRAFÍA: ESTADIOS DE ADOPCIÓN PARA REALIZAR CONTROL MAMOGRÁFICO EN MUJERES DE DISTINTO ORIGEN CULTURAL Y SOCIAL
}

\author{
I HAVE INTENTION TO GET A MAMMOGRAM: STAGES OF ADOPTION FOR \\ MONITORING MAMMOGRAPHY IN WOMEN OF DIFFERENT SOCIAL AND \\ CULTURAL BACKGROUND
}

\author{
Rosa Puigpinós-Riera' ${ }^{1,2,3}$, Mariona Pons-Vigués ${ }^{1,4,5}$, Gemma Serral 1,2,3, \\ $M^{a}$ Dolors Rodríguez-Arjona ${ }^{7}, M^{a}$ Isabel Pasarín ${ }^{1,2,3}$ \\ ' Agència de Salut Pública de Barcelona, Barcelona, España \\ ${ }^{2}$ CIBER de Epidemiología y Salud Pública (CIBERESP), España \\ ${ }^{3}$ Institut d'Investigació Biomèdica Sant Pau (IIB Sant Pau), Barcelona, España \\ 4 Institut Universitari d'Investigació en Atenció Primaria Jordi Gol (IDIAP Jordi Gol), Barcelona, España \\ ${ }^{5}$ Universitat de Girona, Girona, España
}

Resumen

Objetivo: Describir el estadio de adopción en cuanto a la toma de decisión de hacerse controles regulares de cáncer de mama en mujeres de distinto origen sociocultural residentes en Barcelona en el año 2009.

Métodos: Encuesta transversal realizada a una muestra de 960 mujeres de 45 a 69 años residentes en Barcelona en el año 2009 autóctonas e inmigrantes de países en vías de desarrollo. Se desarrolló un cuestionario telefónico. Las variables dependientes fueron los estadios de adopción en que se encontraban las mujeres según el Modelo Transteórico de las etapas de cambio: precontemplación, contemplación, acción, mantenimiento y recaída. Las variables independientes fueron: país de origen, clase social, edad, tiempo de estancia en el país de acogida, conocimiento del idioma, tener hijos, antecedentes familiares de cáncer y nivel de estudios. Se realizó un análisis descriptivo y se estimaron modelos de regresión de Poisson robusta para cada estadio con el fin de obtener las razones de prevalencia según las variables independientes

\section{Abstract}

Objective: To describe the stage of adoption in terms of decision making regular checkups for breast cancer in women of different sociocultural backgrounds living in Barcelona in 2009.

Methods: A cross-sectional survey of a sample of 960 women aged 45 to 69 years living in Barcelona in 2009, native and immigrants from developing countries. We developed a telephone questionnaire. The dependent variables were the stages of adoption in which women were under the Transtheoretical Model: precontemplation, contemplation, action, maintenance and relapse. The independent variables were: Country of origin, social class, age, length of stay in the host country, language skills, have children, family history of cancer and educational level. We performed a descriptive analysis and regression models were estimated robust Poisson each stage in order to obtain the prevalence ratios as independent variables and multivariate models to compare the different stages versus maintenance.

\section{Correspondencia:}


y modelos multivariados para comparar los distintos estadios versus el de mantenimiento.

Resultados: El $\mathbf{9 0} \%$ de las mujeres autóctonas se sitúan en el estadio de mantenimiento, $73 \%$ de las Latinoamericanas, $41 \%$ de las de Europa del Este, $47 \%$ de las chinas (47\%), $58 \%$ de las filipinas, $70 \%$ de las magrebíes y $80 \%$ de las indopakistaníes.

Conclusiones: Es necesario mejorar el conocimiento del cáncer de mama y su prevención trabajando conjuntamente con personas del propio colectivo sobretodo en el caso de las mujeres chinas, filipinas y de Europa del Este.

Palabras clave: Cribado, cáncer de mama, inmigración, estadios de adopción, barreras y beneficios.
Results: Ninety percent of native women are in the maintenance stage, $73 \%$ of Latin American, $41 \%$ of Eastern Europe, $47 \%$ of Chinese $(47 \%), 58 \%$ of the Philippines, $70 \%$ of Maghrebi and $80 \%$ of Pakistani-Indian.

Conclusions: To improve knowledge of breast cancer and its prevention by working with people from the community itself especially in the case of Chinese women, Filipino and Eastern European women.

Keywords: Screening, Breast cancer, migration, stages of adoption, barriers and benefits.

\section{INTRODUCCIÓN}

En España, alrededor del $78 \%$ de las mujeres de 50 a 69 años se hacen mamografías regulares según la Encuesta Nacional de Salud del 2006 (ENS-2006) $^{(1)}$ y lo mismo sucede en Barcelona, en donde según la Encuesta de Salud del 1992 (ESB92) solo un $29,2 \%$ de las mujeres se controlaban de manera regular y en el 2006 (ESB-06) declararon hacerlo el $81 \%$ de las mujeres $^{(2)}$. Los programas de cribado poblacionales han contribuido a este cambio de actitud $^{(3)}$. Estos datos tan optimistas, no deben hacer olvidar que son globales $y$ que siguen existiendo grupos de mujeres, en los que la situación es notablemente distinta. Algunos estudios han mostrado que las mujeres de nivel socioeconómico más bajo participan menos en los programas de cribado aunque esta desigualdad también está influida por otros factores relacionados como el origen cultural, la edad, el entorno y en definitiva todo un complejo entramado de determinantes ${ }^{(4-7)}$ que confluyen e interactúan.

La migración económica y el proceso migratorio en sí mismo, es otro factor o determinante que se añade a los anteriores, y que hace que las personas inmigrantes, sobretodo de inmigración reciente, utilicen menos los servicios preventivos en general $^{(8)}$ y los cuidados vinculados a la mujer, tales como el cribado mamográfico o de cérvix ${ }^{(9)}$, siendo el factor migratorio más decisivo que el de pertenecer a grupos culturales específicos ${ }^{(10)}$.

Sea como sea, son diversos los elementos que juegan un papel relevante a la hora de que las personas adopten un comportamiento en salud, y determinan el estadio en que se encuentran: pueden estar valorando el hacerse una mamografía o simplemente ni tan siquiera piensan en ello. El estadio de conducta en que se encuentren las personas hace que se comporten de modos cualitativamente distintos y además, estos estadios no son compartimentados, si no que son el resultado de unos $\operatorname{procesos}^{(11)}$.

Existen distintos modelos conceptuales para estudiar todo este proceso, si bien el Modelo de Creencias en Salud (Health Belief Model o HBM) y el Modelo Transteórico del Cambio (Transtheoretical Model o TTM), son los dos más utilizados para explicar los comportamientos en cuanto a la realización de mamografías ${ }^{(12,13)}$. Los dos 
establecen que la acción se produce como un equilibrio entre un balance de beneficios y barreras en el HBM y de pros y contras en el TTM. Por otro lado, la percepción de vulnerabilidad o susceptibilidad actúa también en ambos definiendo que la mujer pueda encontrarse en alguno de los estadios de los distintos modelos: precontemplación, contemplación, acción, mantenimiento y recaída ${ }^{(14-17)}$. Además, el TTM ha sido validado para los distintos estadios en los que se puede encontrar una mujer respecto a tomar la decisión de hacerse una mamografía según su origen cultural (filipinas, latinas, afroamericanas $y$ chinas $)^{(18)}$.

Tanto la ESB-2006 como la ENS-2006 mostraron diferencias en cuanto a la realización de prácticas preventivas y controles mamográficos en función del origen cultural de las mujeres y la clase social, siendo la desigualdad de clase social mayor en las mujeres inmigrantes que en las autóctonas $^{(1,19)}$. Por otro lado, la participación de las mujeres inmigrantes en el programa poblacional de cribado de mama de la ciudad de Barcelona era también inferior que en las autóctonass ${ }^{(20,21)}$. El cáncer de mama es una enfermedad grave, que como cualquier otra enfermedad tiene una representación social en las personas en la que confluyen conocimientos del imaginario colectivo, científicos, creencias y valores, elementos todos ellos que van más allá de los factores de riesgo que se le asocian ${ }^{(22)}$. Por otro lado y según fuentes del Institut d'Estadística de Catalunya (IDESCAT), la población de mujeres inmigrantes en edad de población diana del programa, es decir de 50 a 69 años, ha ido aumentado durante los últimos años debido sobretodo a la reagrupación familiar. Por todo ello, es necesario llevar a cabo acciones específicas para favorecer el acceso equitativo a las prácticas preventivas en todas las mujeres. En consecuencia, se consideró importante llevar a cabo un estudio sobre los conocimientos, creencias y percepciones que tenían las mujeres de distinta clase social y origen cultural respecto al cáncer de mama y su prevención secundaria (detección precoz); también conocer el balance entre barreras y facilitadores percibidos para acceder al programa de cribado de la ciudad, y en definitiva en qué estadio se encuentran las mujeres residentes en Barcelona. Esta investigación consta de dos subestudios: uno de ellos con metodología cualitativa y otro con metodología cuantitativa. El objetivo de este trabajo es describir y comparar los estadios de adopción del cribado mamográfico de las mujeres de distintos orígenes socioculturales residentes en Barcelona. Para ello se presenta parte del estudio cuantitativo y se utiliza parte del cualitativo para explicar alguno de los fenómenos observados.

\section{MÉTODO}

\section{Diseño, población de estudio y muestra}

Estudio transversal a partir de una encuesta realizada en Barcelona en el 2009. La población de estudio fueron las mujeres residentes en Barcelona de 45 a 69 años autóctonas o inmigrantes procedentes de países en vías de desarrollo ( $N=253.125$ mujeres). Las mujeres autóctonas se tomaron como grupo de referencia. Los países en vías de desarrollo seleccionados fueron los mismos estudiados en la investigación cualitativa previa( ${ }^{(20,23)}$, escogiendo los países más numerosos y culturalmente diversos de la ciudad. Concretamente, se seleccionaron las mujeres nacidas en las siguientes regiones: Latinoamérica, países de Europa del Este, Magreb, Filipinas, China, Pakistán e India. Se predefinió una muestra de 960 mujeres, distribuidas entre los distintos grupos según su proporción en la población de estudio (278 autóctonas, 257 latinoamericanas, 154 de países de Europa del Este, 96 del Magreb, 78 de 
Filipinas, 75 de China y 25 de Pakistán o India). Una muestra representativa de cada grupo de estudio fue seleccionado mediante muestreo aleatorio simple del padrón de habitantes. Para obtener la muestra, fue necesario contactar con 2.822 mujeres. En promedio, se contactó con 1,6 autóctonas y 3,8 inmigrantes para reclutar una participante de cada grupo. Los principales factores de no participación fueron: falta de voluntad de participar (13,3\%), cambio de domicilio $(39,9 \%)$ e imposibilidad de contactar con la posible participante $(41,6 \%)$. Fue motivo de exclusión tener o haber tenido cáncer de mama $(3,8 \%$ de las no participantes).

\section{Desarrollo del cuestionario de estudio}

Se desarrolló un cuestionario estructurado ${ }^{(24)}$. La mayoría de preguntas fueron extraídas de cuestionarios de estudios previos sobre cribado de cáncer ${ }^{(25-27)}$ y se añadieron nuevas preguntas desarrolladas a partir de la investigación cualitativa pre$\mathrm{via}^{(20,23)}$. El cuestionario fue adaptado por un equipo de investigadores expertos en temas de cribado de cáncer de mama, en inmigración y en desarrollo de cuestionarios. Además, se realizó una prueba pretest a 17 mujeres y una prueba piloto a 50 mujeres de la población de estudio. Se identificaron aspectos a mejorar que fueron introducidos en el cuestionario final. La versión final del cuestionario incluyó 72 preguntas agrupadas en 7 áreas: 1) características demográficas y socioeconómicas, 2) conocimientos sobre el cáncer de mama y su detección precoz, 3) creencias y actitudes hacia la salud y el cáncer de mama, 4) beneficios y barreras percibidas de la mamografía, 5) conductas de detección precoz del cáncer de mama, 6) el programa de cribado de Barcelona, y 7) uso de los servicios sanitarios. La mayoría de preguntas de las áreas 2, 3, 4 y 7 fueron formuladas como frases donde la respuesta era tipo escala de Likert. Este estudio se centra en las áreas 1 y 5 . El resto de áreas se encuentran descritas en otro artículo ${ }^{(28)}$. El cuestionario se desarrolló en catalán y español, y posteriormente, fue traducido al inglés, ruso, árabe y chino. Para asegurar la correcta traducción, una persona bilingüe de cada idioma y experta en temas de salud revisó el cuestionario.

\section{Recogida de datos}

El trabajo de campo duró 5 meses, de diciembre de 2008 a abril de 2009. A las mujeres seleccionadas a través del padrón, se les enviaba una carta, la cual describía el estudio e informaba de que en unos días una encuestadora Ilamaría a su casa. El cuestionario era administrado telefónicamente por mujeres encuestadoras previamente formadas. Las Ilamadas se realizaban de lunes a viernes de 9 de la mañana a 9 de la noche. Se realizaban 9 llamadas en diferentes horarios antes de considerar a una mujer como no participante. Para alcanzar el número de cuestionarios, también se realizó una búsqueda de posibles informantes a través de distintas asociaciones y centros religiosos $(3,8 \%$ de las encuestas realizadas). Las preguntas se contestaban con unos 17 minutos de media. El 13,5\% de las participantes contestaron la encuesta en un idioma distinto al catalán o español debido a que tenían dificultades idiomáticas. El control de calidad de las entrevistas se aseguró mediante formación a las encuestadoras, simulacros y observación directa. El estudio fue aprobado por un comité de ética y la confidencialidad de los datos se aseguró en todas las fases del mismo.

\section{Variables}

\section{Variables dependientes}

Se definieron los siguientes estadios de adopción del cribado mamográfico ${ }^{(15,22)}$ : 
- Precontemplación: no haberse realizado nunca una mamografía y no tener intención de hacerlo en los 2 próximos años.

- Contemplación: no haberse realizado nunca una mamografía y tener intención de hacerlo en los 2 próximos años.

- Acción: haberse realizado una mamografía en los últimos 2 años, pero tener una mamografía anterior a esta y tener intención de realizarse otra mamografía en los próximos 2 años.

- Mantenimiento: haberse realizado una mamografía cada 2 años en los últimos 4 años, la última de las cuales en los últimos 2 años, y tener la intención de realizarse otra en los próximos 2 años.

- Recaída: existen 2 posibles situaciones. 1) haberse realizado una mamografía en los últimos 2 años o 2 mamografías bianuales en los últimos 4 años, pero no tener intención de realizarse más en el futuro. 2) Tener mamografías anteriores, pero no en los últimos 2 años y no tener intención de realizarse más mamografías en los próximos 2 años.

El estadio de adopción de las mamografías se obtuvo de las siguientes preguntas de la encuesta: "¿Se ha hecho alguna vez una mamografía?", “¿Cuántas mamografías se ha hecho?", "¿Cuánto tiempo hace que se realizó la última mamografía?", "¿Cuánto tiempo pasó entre las dos últimas mamografías?", "¿Tiene intención de hacerse una mamografía en los próximos 2 años?".

\section{Variables independentes}

Las variables independientes fueron:

- País de origen: a partir del país de nacimiento de las mujeres, se categorizó en 7 grupos: autóctonas, latinoamericanas, mujeres de países de Europa del Este, magrebíes, filipinas, chinas y pakistaníes e indias.

- Clase social: se distinguieron 2 categorías de clase social: clase manual (IV-V) y clase no manual (I-II-III). La clase social de las mujeres se estableció a partir de la ocupación actual o de la última ocupación de la mujer (y en caso de no existir, a partir de la ocupación de la pareja), ocupación clasificada según la adaptación española del British Registar General propuesta por la Sociedad Española de Epidemiología(29).

- Edad: agrupada en 45 a 49, de 50 a 59 y de 60 a 69 años.

- Medio de procedencia: tipo de área en la cual se ha residido mayor tiempo (urbano o rural). Se consideró área urbana las ciudades de más de 10.000 habitantes, y área rural las poblaciones de menos de 10.000 habitantes.

- Convivencia en pareja: variable dicotómica.

- Tener hijos: variable dicotómica.

- Tener familiares con cáncer de mama: variable dicotómica.

- Nivel de estudios: no leer ni escribir, sin estudios, estudios primarios, secundarios y universitarios.

Y en el caso de las mujeres inmigrantes, también:

- Tiempo de estancia: categorizado en 2 grupos, 10 años o menos y más de 10 años.

- Capacidad de utilizar alguno de los idiomas oficiales de Cataluña: si la mujer es capaz o no de mantener una conversación en español o catalán.

\section{Análisis de los datos}

Se realizó una descripción de todas las variables según origen o procedencia. Se realizó un análisis descriptivo de las distintas frecuencias de los estadios de adopción de la mamografía según origen, clase social, edad, medio de procedencia, convivencia en pareja, tiempo de estancia en el país de acogida, idioma, tener hijos, antecedentes familiares y nivel de estudios. Se estimaron modelos de regresión de Poisson robusta ${ }^{(30)}$ para cada estadio de 
adopción en comparación al estadio de mantenimiento con el fin de obtener las razones de prevalencia ajustadas por edad $(R P)^{(31)}$ según las distintas variables independientes y sus intervalos de confianza al 95\% (IC95\%). En estas regresiones, se juntaron los estadios precontemplación y contemplación. Finalmente, se ajustaron 3 modelos multivariados con el fin de determinar la asociación entre cada estadio de adopción en comparación al estadio mantenimiento con las variables origen, clase social, edad, medio de procedencia convivencia en pareja, tiempo de estancia en el país de acogida e idioma.

En todos los modelos de regresión elaborados, se comprobó la bondad de ajuste a partir de la desviación. En los casos donde había sobredispersión de los datos, se corrigió el modelo con el parámetro $\left(x^{2}\right)$ de sobredispersión ${ }^{(32)}$. Para el análisis estadístico de los datos se utilizó el paquete estadístico STATA/SE 10.1 ${ }^{(33)}$.

\section{RESULTADOS}

En la tabla 1, se describe la muestra de las 960 mujeres encuestadas. En todos los colectivos salvo las autóctonas, chinas e indo pakistaníes predominan las mujeres de clase social manual si bien es en el grupo de las de Europa del Este en el que predominan las mujeres con estudios universitarios. La mayoría proceden de zonas urbanas y las de Europa del Este y América Latina mayoritariamente no viven en pareja y son las que hace menos tiempo que han emigrado (10 años o menos). Las chinas son las que en menor proporción hablan catalán o castellano. La mayoría tienen hijos y entre un $8 \%$ de las chinas y casi un 23\% de las autóctonas dicen tener familiares con cáncer de mama.

En la tabla 2 se puede observar como se distribuyen las mujeres en cada uno de los estadios. Se observa que en el estadio de mantenimiento, que es en el que realmen- te se ubica a las mujeres que llevan a cabo controles de manera regular, se encuentran el $90 \%$ de las autóctonas. En el caso de las inmigrantes la situación cambia, siendo los colectivos de mujeres de Europa del Este $(41 \%)$, las chinas $(47 \%)$ y las filipinas $(58 \%)$ las que menos mujeres tienen en este estadio y en consecuencia los que más mujeres tienen distribuidas entre los otros estadios, entre ellos el de la recaída y precontemplación, que son las que tienen la certeza de no hacerse ninguna mamografía en los próximos 2 años. La mayoría de las mujeres ( $82 \%$ ) que se encuentran en el estadio de mantenimiento pertenecen a las clases sociales altas (no manuales), mientras que esta relación se invierte en los otros estadios. Las mujeres entre $50 \mathrm{y}$ 69 años, que son las de población diana del programa de cribado, también son las que mayoritariamente están en el estadio de mantenimiento al igual que las que provienen de medio urbano. En cuanto a las mujeres inmigrantes, las que hace 10 años o más que están en el país de acogida y las que hablan castellano y/o catalán también se encuentran mayoritariamente en el estadio de mantenimiento.

En la tabla 3, se ven los resultados obtenidos al ajustar modelos bivariados y multivariados con las variables independientes. El grupo de referencia son las mujeres que se encuentran en el estadio de mantenimiento. En cuanto al origen, se puede observar que todos los grupos de mujeres inmigrantes tienen menos probabilidad que las autóctonas de estar en el estadio de mantenimiento. En el de precontemplación y/o contemplación destacan las mujeres de Europa del Este que tienen 25 veces más de probabilidades de estar en ese estadio que las autóctonas; al ajustar por las otras variables independientes estudiadas, esta probabilidad es de 12 veces más $(R P=12,09$ IC95\% 4,51-32,4). Destacan también en este estadio las mujeres chinas que tienen una probabilidad de más de 


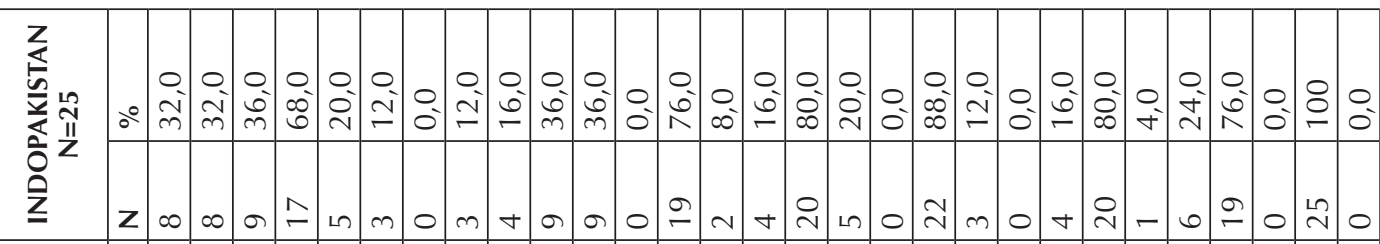

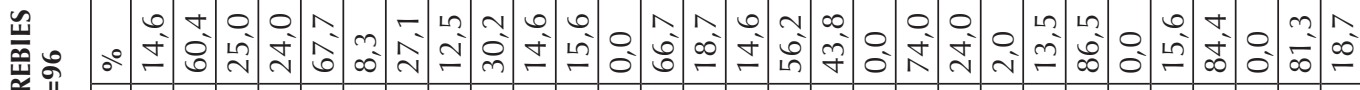
$\sum^{\frac{c}{i}}$

Z

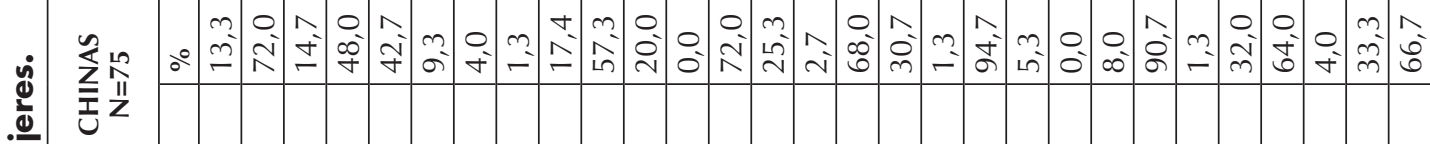

$\mathbf{z}$ 을

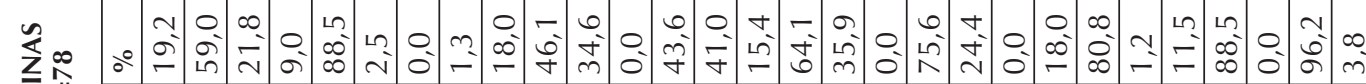
正

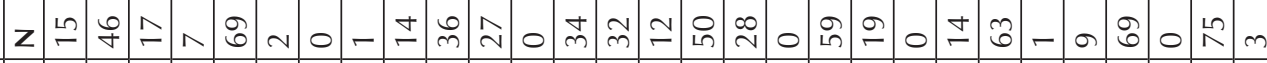

옴

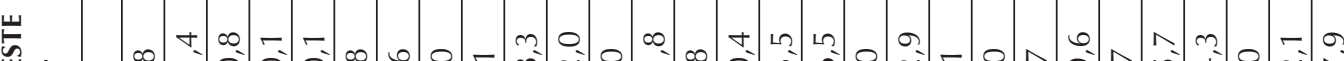

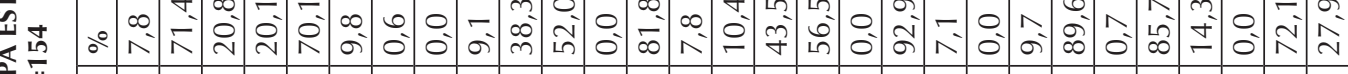

仓ัญ

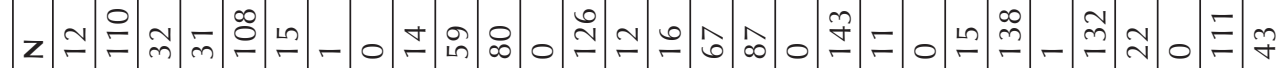

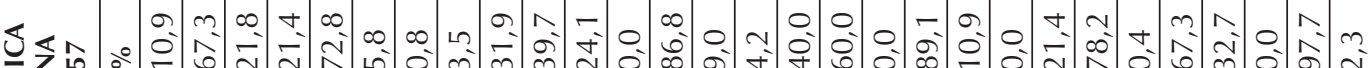

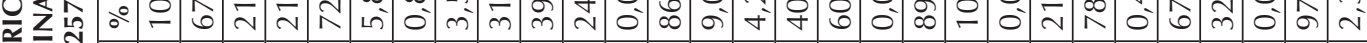
$\sum_{<} \leq \mathbb{Z}$

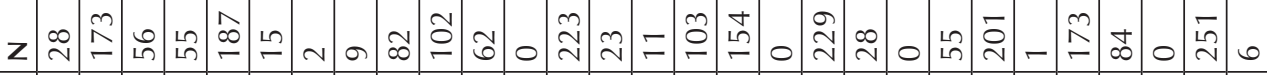

응

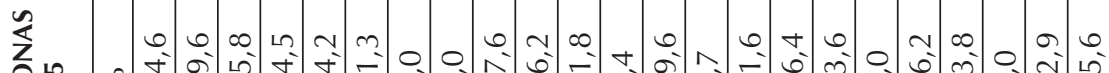

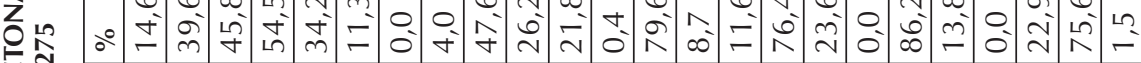

正

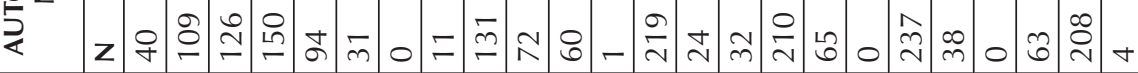

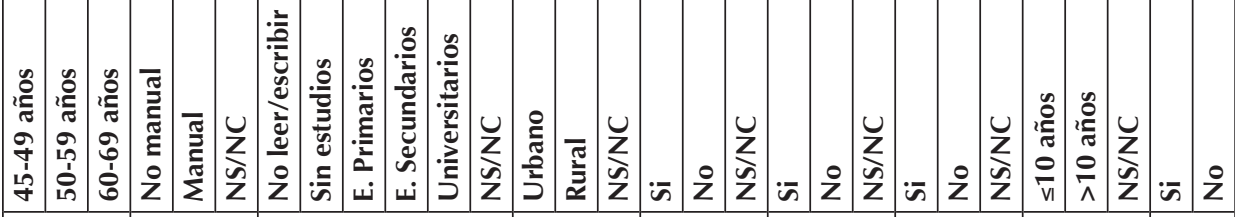

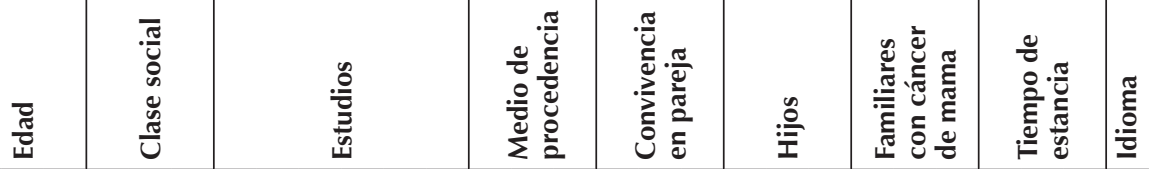




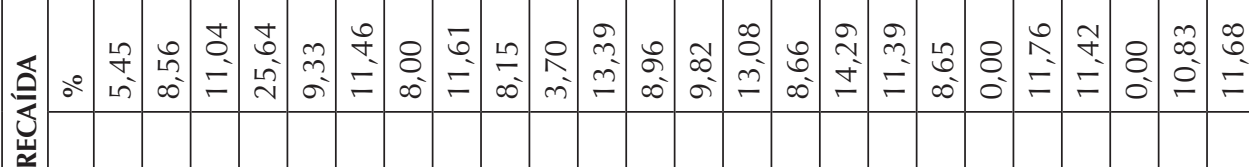

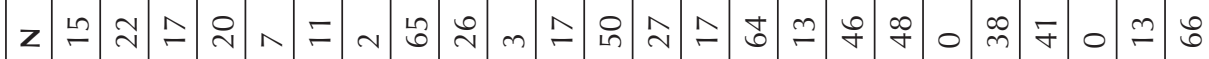

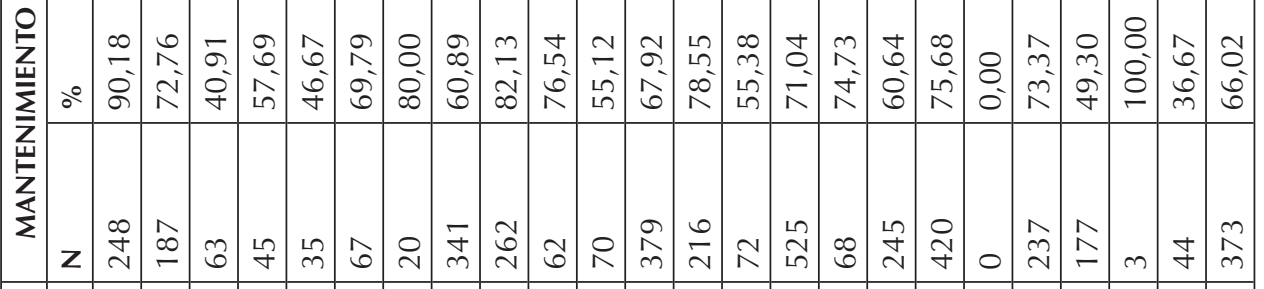

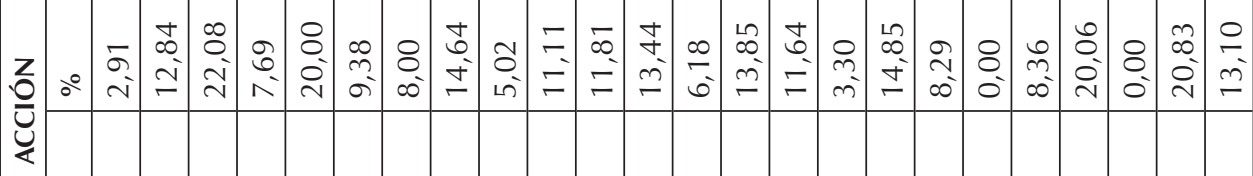

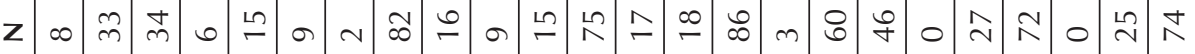

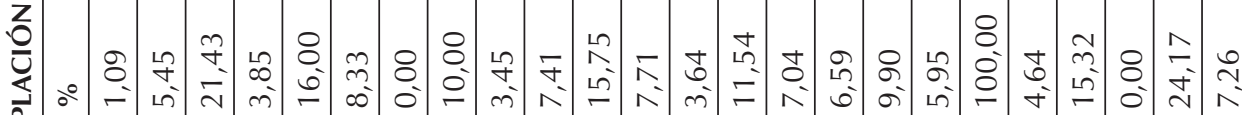

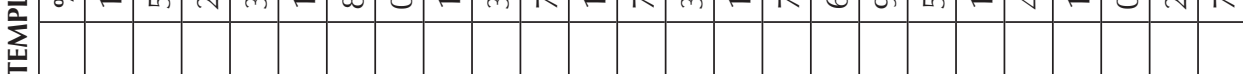

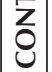

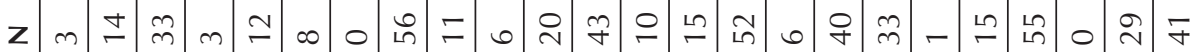

$\underline{z}$

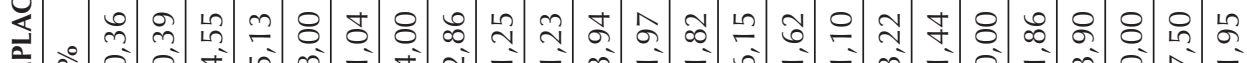

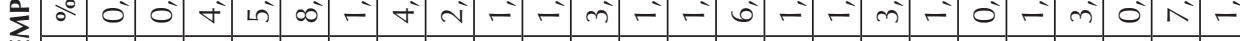

崖

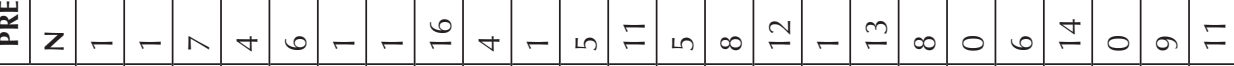

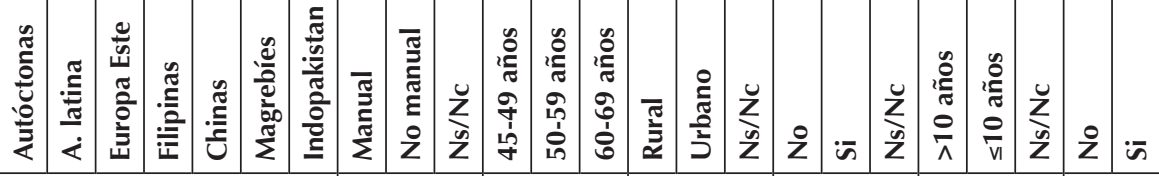

$\overline{\frac{.}{5}}$

离

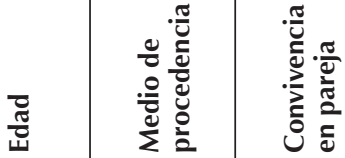

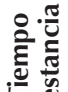


15 veces mayor que las autóctonas de estar en precontemplación/acción ( $R P=15,6$ IC95\% 5,65-43,10). En el estadio de acción a excepción de las filipinas, todos los demás colectivos tienen más probabilidades que las autóctonas de estar en ese estadio destacando nuevamente las chinas y mujeres del Este de Europa. Finalmente, en la recaída, son las mujeres filipinas las que tienen la mayor probabilidad de ubicarse en este estadio, seguidas por mujeres del Este de Europa y chinas. Las mujeres de América latina, magreb e indopakistaníes, tienen una probabilidad no significativa de estar en la recaída.

En cuanto a la desigualdad de clase, se puede observar tanto en los modelos bivariados como en los multivariados, que las mujeres de nivel social alto (no manual), tienen menos probabilidades de estar en los estadios que implican no estar llevando a cabo mamografías regulares $(R P=0,43$ IC $95 \% 0,24-0,74)$. En cuanto a la edad también se puede observar al igual que en el análisis descriptivo que las mujeres de 50 a 69 años son las que mayoritariamente se encuentran en el estadio de mantenimiento, ya que se encuentran en menor proporción en los otros estadios a excepción de la acción en la que esta diferencia no es significativa. Hablar el idioma y el tiempo de permanencia en el país de acogida, no influyen significativamente en el hecho de estar en el estadio de recaída, pero si en estar en los estadios de precontemplacióncontemplación y acción, lo cual significa que las mujeres más integradas en el país de acogida están mayoritariamente en el estadio de mantenimiento, es decir, haciendo controles regulares.

\section{DISCUSIÓN}

El 90\% de las mujeres autóctonas se encuentran en el estadio de mantenimiento de la acción que significa que llevan a cabo controles regulares. Las inmigrantes en menor medida que las autóctonas, aunque con algunas diferencias según colectivos, siendo las de Europa del Este, chinas y filipinas, las que en menor proporción se encuentran en este estadio. Del mismo modo, las mujeres de nivel social más alto, las que tienen entre 50 y 69 años, las que proceden de medio urbano y las que conviven con pareja se encuentran mayoritariamente en el estadio de mantenimiento. En el caso de las inmigrantes, Ilevan a cabo controles regulares de cáncer de mama las que hace más de 10 años que viven en el país de acogida y las que hablan alguna de las lenguas autóctonas.

El estudio cualitativo llevado a cabo ${ }^{(21,23)}$ ayuda a explicar los resultados obtenidos en el trabajo cuantitativo a través de la identificación de las barreras y facilitadores expresados por las mujeres y que van en acorde a resultados observados en estudios similares pero realizados en otros ámbitos. En nuestro estudio pues, chinas, filipinas y mujeres de Europa del Este son las que en menor medida llevan a cabo controles regulares. Vamos a ver, en qué medida las barreras y facilitadores pueden influir en este hecho.

\section{Barreras y facilitadores}

Vivir en pareja ${ }^{(34-36)}$ se ha descrito como un elemento favorable para realizar controles mamográficos y en nuestro estudio se puede observar que las mujeres latinas y las de Europa del Este, son las que menos viven en pareja, cosa que concuerda con el tipo de emigración feminizada que caracteriza estos colectivos y en el caso de las mujeres del Este de Europa solo el $41 \%$ se encuentran en el estadio de mantenimiento.

La barrera idiomática en el colectivo de mujeres chinas es muy importante, ya que son las que en menor proporción hablan alguno de los idiomas del país de acogida, tal como muestran los resultados obtenidos. Si bien más del $64 \%$ dice hacer más 


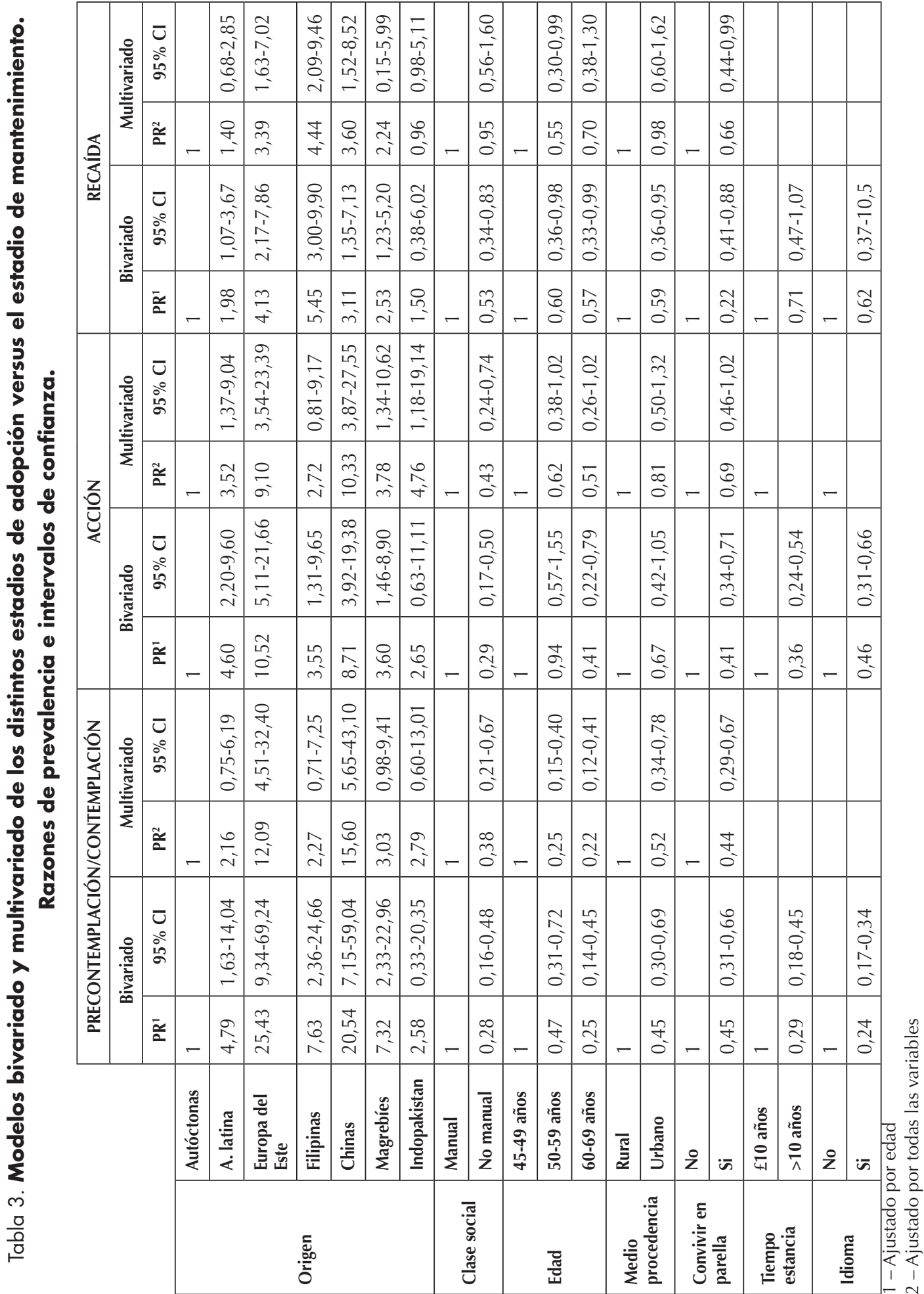


de 10 años que viven en el país de acogida, solo el 33\% declaran ser capaces de hablar alguna de las lenguas autóctonas, lo cual dificulta la integración; otro elemento que puede añadirse a esta dificultad, puede ser el hecho de que es probablemente el único colectivo que más que buscar trabajo en el país de acogida, en cierto sentido, emigran Ilevando consigo su trabajo, estableciendo comercios propios de distinta índole, en los que los empleados son todos chinos, sus clientes en muchas ocasiones son también del mismo colectivo y acaban por tener zonas enteras de la ciudad que constituyen una pequeña china, los tradicionalmente Ilamados "chinatown", tal como se puede observar ya en determinadas zonas de Barcelona del Ensanche principalmente.

Otra barrera detectada en el caso de chinas y filipinas es la gran desconfianza que tienen en el sistema sanitario del país de acogida, prefiriendo en muchos casos aprovechar para hacerse una revisión general cuando viajan a su país:

FP35: "Por las experiencias que han tenido sus amigos o sus amigas. [...] no quiero ir...al hospital, a la urgencia.......] Es que mi amiga se ingresaron, la ingresaron en el hospital y cuando salió ya es un cadáver [...]". (Mujer filipina).

XsY23: "Si, hay muchos médicos son practicantes, lo que yo sepa, eh. Porque yo he visto amigo, amiga hicieron operación mal, hay que operar otra vez. Más grave, muy mal, eh. Por ejemplo, yo tengo una señora que trabajaba en una compañía de seguros. Ella tenía un hongo aquí, un hongo aquí detrás de la [...] entonces hizo operación, torcido la boca, torcido ojo, ahora todo torcido, ahora todo torcido, toda la cara torcida [...]". (Mujer china).

Hay también en muchas ocasiones recelo y desconfianza hacia la medicina occi- dental. Si bien le reconocen el papel que tiene en el caso de enfermedades graves como el cáncer, en general para las enfermedades comunes prefieren la medicina propia.

XsY23: "Pastillas aquí muy mal. Porque ya sabemos, hacemos algo aquí en España, la gente come pastillas como comer". (Mujer china).

Otra barrera importante, sobretodo en el colectivo de mujeres chinas, es el miedo que les infunde el cáncer y que hace que incluso de hablar de la enfermedad sea un tabú; les provoca mucha tristeza y para la cultura china la alegría es el motor de la vida:

XsY23: "Dice que hay gente que no le gusta escuchar sobre este tipo de enfermedad, que hay gente, en China, que no quiere escuchar de este tipo de enfermedad. Porque esta enfermedad es muy triste [...]. En los chinos cáncer es enfermedad muy triste. Españoles diferente [...] mucho, porque la vida muy corta. Hay mucha gente española...[...] hay gente que está "no importa", relajar mucho, no pasa nada. Pero también, yo si tengo cáncer no puedo estar tranquila, de verdad, eh?" (Mujer china).

Además son unos colectivos que de manera muy marcada priorizan el trabajo por encima de las demás necesidades.

XaH28: "Porque aquí todo el mundo está trabajando, la gente muy ocupada [...] menos tiempo. En China,..., yo creo en China es diferente... Ellas se cuidan más que nosotros. Porque ellas en China trabajan muy ligero ellos. Aquí no hay,..., Aquí cada día estás trabajando, ¿no? En China, chinas no tanto trabajo, pero se vive muy bien mañana y noche."(Mujer china).

FP35: "Es porque la prioridad de los filipinos es ganar dinero. Están aquí para 
ganar dinero y para poder ayudar a los niños de la familia que han quedado en Filipinas. Y también para sus hijos que están aquí en, en España ¿no? Si no trabajan no pueden vivir aquí. Y también, tampoco sus familias en Filipinas". (Mujer filipina)

En las mujeres del Este, sobretodo las que provienen de las zonas industriales tienen muy arraigado el sentido de las revisiones laborales impuestas por los regímenes comunistas y que les inculcaban el hecho de que hay que estar sano para trabajar.

EAM21: "Las mujeres vienen aquí para trabajar, que la mayoría vienen para trabajar, no para divertirse, vienen.....son mujeres sanas. Porque antes de marcharse del país siempre se hacen una revisión. Para empezar a trabajar, la mentalidad es que tienes que estar sana, ¿no? Entonces ellas vienen con un control muy bien hecho para empezar a trabajar". (Mujer del este de Europa).

A pesar de ello, una vez en el país de acogida, y a pesar de la necesidad resentida de estar sanas, descuidan su propia salud porque tienen otras prioridades, sobretodo las que tienen hijos y han emigrado solas, ya que sienten que toda la responsabilidad de salir adelante recae sobre ellas.

EAT20: "Más creo, responsables madres que tienen niños menor de edad. Porque saben, sobre todo si es madre soltera, sabe que tiene que cuidarse porque, si le pasa alfo, el niño se queda solo. $Y$ aquí en este país es más complicado porque tienes doble responsabilidad. [...] Ilegan aquí con los niños y no tienen familiares." (Mujer del este de Europa).

Los resultados encontrados están en sintonía con otros estudios; así, en Michigan donde la comunidad asiática tiene una fuerte implementación, encuentran que son también chinas y filipinas las que en menor proporción se encuentran en el estado de mantenimiento hallándose principalmente en el de precontemplación ${ }^{(37)}$. Esto es así por el desequilibrio entre los cons (barreras) y los pros (beneficios percibidos), siendo las principales barreras, de tipo logístico (tener un seguro, accesibilidad al centro, dificultades idiomáticas), de proceso (dolor, miedo a radiaciones innecesarias, tener que desnudarse), aunque en las mujeres asiáticas de nivel social alto que disponen todas ellas de un seguro este equilibrio cambia ${ }^{(38)}$, problema que en nuestro ámbito debido a la existencia de un servicio nacional de salud y el acceso que a él tienen los inmigrantes, este factor no influye en la misma medida.

Mediadora: "Dice PH2 que, como en Pakistán hay que pagar todas las pruebas, y la gente no le gusta ir. Porque en mi país la mayoría son pobres y no pueden gastar tanta pasta. $Y$ aquí, las mujeres, como el sistema sanitario es totalmente gratis,..., pues van ha hacerse las pruebas." (Mujer pakistaní).

Otro estudio realizado con mujeres camboyanas de Seattle, muestra como factores decisivos para estar en el estado de mantenimiento, el sexo del médico, tener seguro médico, pensar que el cáncer de mama se puede curar y llevar 10 años o más en Estados Unidos, siendo la variable de más peso de todas ellas el sexo del médico. Claramente, las mujeres cuyo médico es una mujer de origen asiático tienen significativamente más probabilidad de estar en el estadio de mantenimiento ${ }^{(39)}$. En otro estudio que implica a mujeres de distintos estados, tener 65 años o más, la situación laboral, vivir en pareja y el nivel de estudios se manifiestan también como variables decisivas, siendo el consejo médico la más relevante para inclinar el balance hacia llevar a cabo la acción 
independientemente del estadio en el que se encuentre la mujer ${ }^{(4))}$. El nivel de conocimiento sobre el cáncer de mama y la probabilidad de curación a través de la detección precoz es también un elemento clave. Aun así, un estudio llevado a cabo con mujeres de edad más avanzada, se muestra que a partir de los 75 años a pesar de tener este conocimiento, las mujeres están menos predispuestas a seguir realizando controles regulares ${ }^{(41)}$, probablemente influenciado también por el hecho de que incluso la mayoría de programas poblacionales de cribado citan las mujeres hasta los 69 años.

Distinguir el estadio exacto en que se encuentran las mujeres así como cuales son las barreras en cada estadio, permite la planificación concreta de actividades; así, el miedo al cáncer de mama, las recomendaciones del médico y hablar el idioma del país de acogida con fluidez (en cuanto a variable indicativa del grado de integración en la sociedad de acogida), son factores clave en el caso de las mujeres que no tienen intención de hacerse una mamografía en un futuro ${ }^{(42)}$. La actitud positiva hacia la prevención es también un factor importante y que puede diferir en función del origen cultural de las personas y el nivel cultural, tal como han mostrado estudios previos con población afroamericana comparada con las mujeres de origen caucásico ${ }^{(43)}$. Des del programa de detección precoz de cáncer de mama de Barcelona, se ha revisado y ampliado la información sobre la enfermedad y el programa que se pone a disposición de las mujeres desde distintas vías: sesiones informativas, páginas web, folletos informativos. Paralelamente, se está trabajando con los profesionales de atención primaria los cuales tienen un papel importante y decisivo aconsejando y acompañando a las mujeres en la toma de una decisión; así, se están reforzando los mecanismos de coordinación entre programa y profe- sionales a fin de reforzar la información de pros y contras vinculados a los programas de cribado que hay que transmitir a la población.

En el caso de las mujeres inmigrantes, el tiempo de permanencia en el país de acogida es importante por diversos motivos: una vez que las mujeres ya se sienten establecidas disponen de más tiempo para curar de la propia salud. Además, han tenido más tiempo para conocer el funcionamiento del sistema sanitario e ir integrando la prevención como un elemento importante a tener en cuenta. En la mayoría de los países de origen de las mujeres encuestadas, la atención sanitaria es escasa y muy privatizada; ello implica que realmente la salud es cuestión de presencia o ausencia de enfermedad y el concepto de la prevención y el acceso universal son conceptos que van integrando con el tiempo y que hace que incluso vayan cambiando el sentimiento que tienen hacia el cáncer de mama. Para muchas de ellas, debido a las vivencias en el país de origen, es una enfermedad que irremediablemente conduce a la muerte y es con el tiempo que van integrando el concepto de prevención y la posibilidad de curación.

FP35: "Dicen que es la enfermedad de los ricos porque hay que tener mucho dinero para superarla". (Mujer filipina)

EG8: "Y yo creo que si tuviéramos estas posibilidades en nuestros países habría muchos menos casos de muertes de mujeres por el cáncer de mama". (Mujer del este de Europa).

\section{LIMITACIONES Y APORTACIONES}

La principal limitación es que a pesar de encuestar a un amplio número de mujeres obtenidas a través de muestreo y haber trabajado además con mediadoras culturales que facilitaban la localización 
de las mujeres para completar la muestra, probablemente no se pudo llegar a las mujeres que estaban en situación más vulnerable y que en consecuencia realizan menos controles preventivos. Por otro lado, los resultados obtenidos en el caso de las mujeres indo pakistaníes pueden estar sesgados debido al bajo número de mujeres encuestadas, aunque cabe explicar que se contactó con todas las mujeres de este origen y de 45 a 69 años empadronadas en la ciudad.

La principal aportación de este trabajo es que es el primer estudio de este tipo realizado en nuestro país y aporta información relevante para mejorar los mecanismos de sensibilización, educación y comunicación en referencia al control regular del cáncer de mama.

\section{CONCLUSIONES Y RECOMENDACIONES}

En los estudios revisados, recibir consejo médico, tener un seguro médico y llevar largo tiempo en el país de acogida, son factores que favorecen la realización de mamografías regulares. En nuestro contexto, existe un servicio nacional de salud y el programa de detección precoz de cáncer de mama convoca a todas las mujeres y busca los mecanismos necesarios para que el mensaje y la información lleguen a todas las mujeres por igual. Chinas, filipinas y mujeres del este de Europa son las que se encuentran en menor medida en el estadio de mantenimiento, distribuyéndose entre la recaída y la acción. Algunas de las principales barreras son difíciles de abordar desde los servicios de salud; estos han de incidir principalmente en ampliar el conocimiento sobre la enfermedad y la prevención. En el caso de las mujeres chinas sobretodo, habría que llevar a cabo acciones específicas con personal autóctono para mejorar el nivel de comprensión y conocimiento mutuos.

\section{AGRADECIMIENTOS}

Los autores agradecen a Anna Manzanera Martorell, Anna Marí Dell'Olmo, Maite López San Pedro, Tatiana Averkina, Mi Zhou and Kenza Chouafa que han participado en la recolección de datos de este trabajo y a todas las mujeres que han participado en él aportando toda la información.

Este estudio ha sido financiado por el "Fondo de Investigaciones Sanitarias, Instituto de Salud Carlos III" (PI061130).

\section{REFERENCIAS BIBLIOGRÁFICAS}

1. Pons-Vigués $M$, Puigpinós-Riera $R$, Rodríguez-Sanz M, Serral G, Palència L, Borrell C. Preventative control of breast and cervical cancer in immigrant and native women in Spain: The role of country of origin and social class. Int J Health Serv 2011;41(3):483-99 Doi:10.2190/ HS.41.3.e

2. Puigpinós-Riera R, Serral G, Pons-Vigués $M$, Palència $L$, Rodríguez-Sanz $M$, Borrell C. Evolution of inequalities in breast and cervical cancer screening in Barcelona: population surveys 1992, 2001, and 2006. J Womens Health (Larchmt). 2011;20(11):1721-7. Doi:10.1089/ jwh.2010.2478

3. Palència $L$, Espelt $A$, Rodríguez-Sanz $M$, Puigpinós R, Pons-Vigués $M$, Pasarín MI, et al. Socio-economic inequalities in breast and cervical cancer screening practices in Europe: influence of the type of screening program. Int J Epidemiol. 2010;39(3):75765. Doi:10.1093/ije/dyq003

4. Jaeyoung K, Soon-Nang J. Socioeconomic disparities in breast cancer screening among US women: Trends from 20002005. J Prev Med Public Health 2008; 41(3): 186-94.

5. Polasek O, Kolcic I, Voncina L, Strnad M, Vuletic S, Kern J. Breast, colon and pros- 
tate screening in the adult population of Croatia: does rural origin matter? Rural Remote Health. [En línea] 2007; 749. [Acceso 10 de abril de 2012] Disponible en: http://ww.rrh.org.au

6. Cabeza E, Esteva M, Pujol A, Thomas V, Sánchez-Contador C. Social disparities in breast cancer preventive practices. Eur J Cancer Prev 2007; 16: 372-9. Doi:10.1097/01.cej.0000236243.55866. b0

7. Dailey AB, Kasl SV, Holford ThR, Calvocoressi L, Jones BA. Neighborhood-level Socieconomic Predictors of nonadherence to mammography screening guidelines. Cancer Epidemiol Biomarkers Prev 2007; 16(11): 2293-303. Doi:10.1158/10559965.EPI-06-1076

8. Pylyochuk Y, Hudson J. Immigrants and the use of preventive care in the United States. Health Econ 2009; 18(7): 783-806.

9. Lofters AK, Moineddin R, Hwang SW, Glazier RH. Low rates of cervical cancer screening among urban immigrants. Med Care 2010; 48(7): 611-8. Doi:10.1097/ MLR.0b013e3181d6886f

10. Sanghavi M, Wee ChC, McCarthy EP, Davis RB, Ngo-Metzger Q, Phillips RS. Racial and ethnic disparities in cancer screening. JGIM 2003; 18:1028-35

11. Durà- Ferrandis E, Andreu-Vaillo Y, Galdón-Garrido MJ. Aplicación de modelos sociocognitivos a la prevención secundaria del cáncer de mama. Psicol Conduct 2001; 9(1): 99-130.

12. Dura E, Andreu Y, Galdón MJ, Tuells J. Razones de no asistencia a un programa de cribado. Psicooncología 2004; 1(1): 3150.

13. Galdón MJ, Durà E, Andreu Y, Tuells J, Ibáñez E. La detección temprana del cáncer de mama: creencias de salud y cribado mamográfico. Psicooncología 2003; 25(1): 8-22.

14. Maxwell CJ, Onysko J, Bancej ChM, Nichol M, Rakowski W. The distribution and predictive validity of the stages of change for mammography adoption among Canadian women. Prev Med 2006; 43: 171-7. Doi:10.1016/j.ypmed.2006.04.018

15. Russell KM, Champion VL, Perkins SM. Development of cultural belief scales for mammography screening. Oncol Nurs Forum 2003; 30 (4): 633-40. Doi:10.1188/03. ONF.633-640

16. Champion VL, Slinner CS. Differences in perceptions of risk, benefits and barriers by stage of mammography adoption. J Womens Health (Larchmt) 2003; 12 (3): 27786. Doi:10.1089/154099903321667618

17. Chamot E, Charvet AI, Perneger TV. Predicting stages of adoption of mammography screening in a general population. Eur J Cancer 2001; 37: 1869-77. Doi:10.1016/ S0959-8049(01)00234-9

18. Otero-Sabogal R, Stewart S, Shema SJ, Pasick R. Ethnic Differences in Decisional Balance and Stages of Mammography Adoption. Health Educ Behav 2007; 34(2): 278-96. Doi:10.1177/1090198105277854

19. La salud de la població immigrant de Barcelona. Barcelona: Agència de Salut Pública de Barcelona, 2008.

20. Agència de Salut Pública de Barcelona. Butlletí Informatiu de l'Oficina Tècnica del Programa de Detecció Precoz de Càncer de Mama de Barcelona (Barcelona) 4. [En línea] Agència de Salut Pública de Barcelona, 2009. [Acceso 30 de abril de 2012] Disponible en: www.aspb.cat/cancer-mama

21. Agència de Salut Pública de Barcelona. Butlletí Informatiu de l'Oficina Tècnica del Programa de Detecció Precoz de Càncer de Mama de Barcelona (Barcelona) 5. [En línea] Agència de Salut Pública de Barcelona, 2009. [Acceso 30 de abril de 2012] Disponible en: www.aspb.cat/cancer-mama

22. Giraldo-Mora CV. Persistencia de las representaciones sociales del cáncer de mama. Rev Salud Pública (Bogota) 2009; 11(4): 514-25. Doi:10.1590/S012400642009000400003 
23. Pons-Vigués $M$, Puigpinós $R$, Rodriguez D, Fernandez de Sanmamed MJ. Estrategias para reclutar mujeres inmigrantes para participar en una investigación cualitativa. Gac.Sanit. 2009;23 Suppl 1:90-2. Doi:10.1016/j.gaceta.2009.07.006

24. Agència de Salud Pública. Detecció precoç del càncer de mama. Altres documents tecnis del programa. Enquesta a dones immigrants sobre el programa de cribratge (annex) [En línea] [Acceso $10 \mathrm{de}$ abril de 2012]. Disponible en: http://www. aspb.cat/cancer-mama

25. Andreu Y, Galdón M, Durá E, García V, Ibáñez E. Participación en cribados mamográficos y creencias de salud: una perspectiva de proceso. Psicooncologia 2007;4(2-3):345-65.

26. Galdón M, Durá E, Andreu Y, Tuells J. Creencias de salud relacionadas con la participación en un programa de cribado mamográfico. Psicol Conduct 2000;8(2):357-73.

27. Galdón M, Durá E, Andreu Y, Queipo S, Ibáñez E. Health beliefs and breast cancer screening: applying the stage-of-change models. Inter J Cancer Prev 2007;3(12):9-28.

28. Pons-Vigués $M$, Puigpinós-Riera $R$, Serral G, Pasarín MI, Rodríguez D, Pérez G, et al. Knowledge, attitude and perceptions of breast cancer screening among native and immigrant women in Barcelona, Spain. Psychooncology 2011. Doi: 10.1002/ pon. 1940

29. Domingo-Salvany A, Regidor E, Alonso J, Alvarez-Dardet C. Una propuesta de medida de la clase social. Grupo de trabajo de la SEE y de la SEMFC. Aten Primaria 2000; 25:350-63.

30. Zou G. A modified poisson regression approach to prospective studies with binary data. Am J Epidemiol 2004; 159: 702-06. Doi:10.1093/aje/kwh090

31. Thompson ML, Myers JE, Kriebel D. Prevalence odds ratio or prevalence ratio in the analysis of cross sectional data: What is to be done? Occup Environ Med 1998; 55: 272-7.

32. Tobías A. Extensión del modelo de regresión con el paquete estadístico STATA. Departamento de Estadística y Econometría, Universidad Carlos III de Madrid: 2005.

33. StataCorp. 2009. Stata Statistical Software: Stata/SE 10.1 for Windows. College Station, TX: StataCorp LP.

34. von Euler-Chelpin M, Olsen AH, Njor S, Vejborg I, Schwartz W, Lynge E. Sociodemographic determinants of participation in mammography screening. Int J Cancer 2008;122 (2):418-23. Doi:10.1002/ ijc.23089

35. Martin-Lopez R, Hernandez-Barrera V, De Andres AL, Garrido PC, De Miguel AG, Garcia RJ. Breast and cervical cancer screening in Spain and predictors of adherence. Eur J Cancer Prev 2010; 19(3):23945. Doi:10.1097/CEJ.0b013e3283372125

36. Lagerlund M, Sparen P, Thurfjell E, Ekbom A, Lambe M. Predictors of nonattendance in a population-based mammography screening programme; sociodemographic factors and aspects of health behavior. Eur J Cancer Prev 2000;9(1):2533. Doi:10.1097/00008469-20000200000004

37. Wu TY, Hsieh HF, West BT. Stages of mammography adoption in Asian American women. Health Educ Res 2009; 24: 74859. Doi:10.1093/her/cyp009

38. Wu TY, West BT. Mammography stage of adoption and decision balance among asian indian and filipino american women. Cancer Nursing 2007; 30 (5): 390-8. Doi:10.1097/01. NCC.0000290812.14571.2C

39. Shing-Ping T, Yasui Y, Kuniyuki A, Schwartz SM, Jackson JC, Taylos VM. Breast cancer screening: Stages of adoption among Cambodian American women. Cancer Detec Prev 2002; 26(1): 33-41.

40. Stoddard AM, Rimer BK, Lane D, Fos SA, Lipkus I, Luckmann R, et al. Underusers 
of mammogram screening: Stage of adoption in five U.S. subpopulations. Prev Med 1998; 27: 478-87. Doi:10.1006/ pmed.1998.0310

41. Skinner CS, Arfken CL, Sykes RK. Knowledge, perceptions and mammography stage of adoption among older urban women. Am J Prev Med 1998; 14(1): 54-63. Doi:10.1016/S07493797(97)00008-1

42. Strong C, Liang W. Relationships between decisional balance and stage of adoption mammography and pap testing among chinese american women. Cancer Epidemiol 2009;33(5):374-80. Doi:10.1016/j. canep.2009.10.002

43. Russell KM, Monahan P, Wagle A, Champion $\mathrm{V}$. Differences in health and cultural beliefs by stage of mammography screening adoption in african american women. Cancer 2007;109(2 Suppl):386-95 
